\title{
Sibylle Laurischk MdB, Rechtsanwältin
}

\author{
Juristinnen machen Karriere - wir stellen sie vor
}

\author{
Das Interview führte Dr. Maria Wersig am 24. Juni 2013.
}

Selbständige Rechtsanwältin, Kommunal- und Bundespolitikerin, alleinerziehend - Frau Laurischk, wie haben Sie es geschafft, Ihre verschiedenen Rollen mit einander zu vereinbaren?

Ich glaube, indem ich mir nicht so furchtbar viele Gedanken darüber gemacht habe, ob das zu leisten ist oder nicht, sondern einfach in die Aufgaben reingegangen bin. Ich habe die Dinge immer mit einer gewissen Begeisterung angepackt, das hat mir geholfen.

Sie haben sich nach der Tätigkeit in einer internationalen Wirtschaftsprüfungskanzlei Anfang der 1980er Jahre für die Selbständigkeit entschieden - warum?

Ich habe zunächst eher fachfremd gearbeitet, in einem betriebswirtschaftlichen Rahmen. Das war fast wie eine zweite Ausbildung für mich. Ich habe damals meinen Mann in dem Unternehmen kennengelernt und mit der Entscheidung für eine Familiengründung wollten wir nicht in Frankfurt bleiben. Wir haben uns dann für den Umzug in meine Heimatstadt Offenburg entschieden, wo ich geboren und aufgewachsen bin. Da war aber die Möglichkeit, in der Wirtschaftsprüfung weiterzuarbeiten nicht gegeben. Ich wollte auch meine Kinder versorgen, habe also in gewisser Weise auch noch dieses klassische Rollenverständnis gelebt. Ich war aber überzeugt davon, dass ich meinen Beruf nicht völlig aufgeben will. Dadurch kam dann der Schritt in die Selbständigkeit. Ich war aber auch vorher schon als Rechtsanwältin in Darmstadt zugelassen. In dem Wirtschaftsprüfungsunternehmen, das war damals einer der sogenannten „Big 8“, wurde Wert darauf gelegt, wenn möglich die Zulassung als Rechtsanwältin zu haben. Aber eigentlich war es im Bereich der Wirtschaftsprüfung damals untypisch, als Juristin tätig zu sein, es war schon ein betriebswirtschaftlich dominierter Bereich. Aber auch Philologen habe ich dort erlebt, Naturwissenschaftler... Als Jurist war ich fast eine Einzelkämpferin.

\section{Haben Sie das dann fortführen können in Ihrer Kanzlei?}

In einer kleinen bis mittleren Stadt wie Offenburg ist das Feld nicht so wie in der Großstadt für diese spezifische Tätigkeit. Ich bin dann in das Familienrecht gegangen, beziehungsweise reingerutscht. Mich hat auch die Frauenbewegung immer sehr getragen, die Problematik von Frauen in Trennung, Scheidung und in Gewaltsituationen hat mich sehr beschäftigt. Ich habe damals auch das Frauenhaus mit aufgebaut und gefördert in Offenburg. Was mir aber immer sehr geholfen hat - ich konnte Bankverhandlungen gut führen. Weil ich in der Wirtschaftsprüfung Banken geprüft habe, wusste ich einfach, wie Banken funktionieren und worauf man achten muss. Das war sicherlich hilfreich für Verhandlungen in vermögensrechtlichen Fragen.
Sibylle Laurischk (evangelisch, geschieden, 3 Kinder) wurde am 12. Dezember 1954 in Offenburg/Ortenaukreis geboren. Nach dem Abitur 1973 in Offenburg studierte sie Rechtswissenschaften an der Universität Heidelberg. 1978 bestand sie das Erste, 1981 das Zweite Juristische Staatsexamen. Von 1981 bis 1983 arbeitete sie in einer internationalen Wirtschaftsprüfungsgesellschaft; seit 1983 ist sie selbstständige Rechtsanwältin in Offenburg in eigener Kanzlei.

1990 trat sie in die FDP ein. Seit 1994 ist sie Stadträtin im Stadtrat Offenburg, seit 1999 Fraktionsvorsitzende. Von 2004 bis 2009 war sie Kreisrätin im Kreistag Ortenau. Sie war von 1998 bis 2012 Stellvertretende Vorsitzende des FDP-Bezirkverbandes Südbaden und ist seit 2003 Stellvertretende Vorsitzende der Liberalen Frauen BadenWürttemberg. Von 2003 bis 2010 war sie FDP-Kreisvorsitzende im Ortenaukreis, von 2006 bis 2008 Bundesvorsitzende der Liberalen Frauen.

2002, 2005 und 2009 wurde sie in den Deutschen Bundestag gewählt. Sie ist gegenwärtig Vorsitzende des Ausschusses für Familie, Senioren, Frauen und Jugend, stellvertretendes Mitglied im Rechtsausschuss und seit September 2011 Mitglied des Vorstands der FDPBundestagsfraktion.

Außerdem ist sie Mitglied im Bundesvorstand Liberale Frauen e.V., im Akademikerinnenbund, Vorstandsmitglied Aufschrei! e.V. und Mitglied bei Astern e.V. Offenburg. Dem djb trat sie 2003 bei.

Sie sind 1990 der FDP beigetreten und in die Politik gegangen. Was waren Ihre Beweggründe dafür?

Die deutsche Einheit. Ich habe die Teilung Deutschlands familiär erlebt, ich erinnere mich sehr gut an den Bau der Mauer, als ich sechs Jahre alt war. Ich war einen Tag vor dem Mauerbau noch als Kind mit meiner Familie zu einem Besuch in der DDR und kann mich noch sehr gut daran erinnern, bis hin zu der starken Verunsicherung meiner Eltern und wie angstbesetzt diese Reise in die DDR damals für meine Mutter war. Meine Familie hat die Teilung und Trennung also sehr direkt erlebt und diese Ungerechtigkeit, der Schießbefehl an der innerdeutschen Grenze, all das hat mich schon sehr geprägt. Ich fand es völlig absurd, wie ein politisches System behaupten konnte, im Interesse der Menschen ihre Freizügigkeit und ihr Leben nicht mehr achten zu müssen. Wenn ich es jetzt zurückblickend betrachte, hat mich das auch belastet. Es war eine Auswirkung des Krieges und insofern bin ich eben auch ein „Nachkriegskind“. Es war für mich sehr bewegend, die Demonstrationen zu erleben und zu sehen, wie die Leute sich das einfach nicht mehr gefallen lassen. Ich hatte damals sehr kleine Kinder, deshalb konnte ich es nur am Fernseher verfolgen, habe diese Zeit aber noch sehr gut in Erinnerung. Das war für mich ein Grund, ein politisches Bekenntnis abzugeben und in die Partei der Frei- 
heit einzutreten. Das war im Januar 1990. Ich hatte aber auch vorher schon einmal kommunal für die FDP kandidiert, war damals aber noch ohne Parteibindung, als freie Kandidatin.

\section{Sie sind dann nach ihrem Eintritt in die FDP relativ schnell in den Bundestag gewählt worden.}

Nein so schnell geht das nicht. Ich hatte ja 1988 schon für die Kommunalwahl kandidiert, war aber nicht gewählt worden. Die Parteileute haben ja Erfahrungswerte, wer Potenzial hat und man hat mich dann gefördert. Auf der kommunalen Ebene lernt man das kleine Einmaleins. Ich hatte ja damals drei kleine Kinder und habe parallel immer gearbeitet und habe dann im Rahmen des Möglichen auch Aufgaben in der Partei übernommen. Ich kann mich zum Beispiel daran erinnern, wie ich abends mit den Kindern unterwegs war und Parteieinladungen in den Briefkästen verteilt habe. Und 1994 bin ich dann mit 40 Jahren in den Stadtrat gewählt worden. Das war also nicht es war wirklich ein sehr spartanischer Wahlkampf. Ich habe aber damals auch bei der Listenaufstellung schon einen guten Platz erreichen können und das war dann auch die Empfehlung für die Wahl 2002. Ich habe bei der nächsten Listenaufstellung auch wieder gegen einen Mann kandidiert und mich durchgesetzt. Ich war dann auf dem aussichtsreichen Listenplatz 6, das war damals so etwas wie der Frauenplatz der FDP in BadenWürttemberg. Das hat geklappt, dann war ich im Bundestag.

\section{Im Bundestag haben Sie ja dann auch die Familienpolitik und die Rechtspolitik zu Ihren Schwerpunkten gemacht.}

Weil ich nicht die klassische Parteikarriere gemacht habe, war ich thematisch gar nicht so festgelegt. Ich habe das auch etwas auf mich zukommen lassen. Aber um auch beruflich etwas über den Tellerrand zu schauen und mich auf dem Laufenden zu halten, hatte ich mich innerhalb der Partei im Landesfachausschuss Innen und Recht engagiert. Das

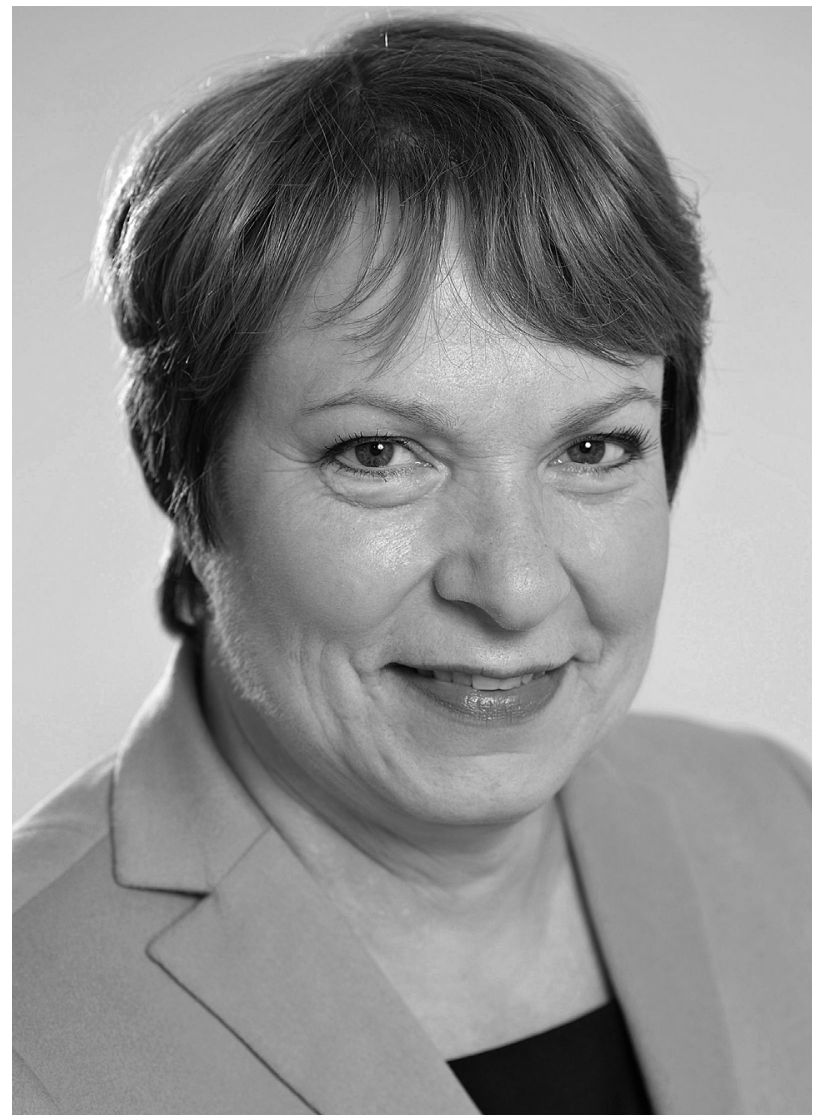

die typische Parteikarriere, die man so ab dem Abitur plant, wie das heute üblich ist. Ich bin eigentlich eine Quereinsteigerin. Ich habe dann aber als Stadträtin einiges bewegen können und habe so auch innerhalb der Partei auf mich aufmerksam gemacht. Ich bin dann 1997 sehr kurzfristig angesprochen worden, ob ich nicht bei der Aufstellung für die Bundestagswahl kandidieren will. Da dachte ich, das mache ich mal. Man glaubt das nicht, aber es war so. Ich war nicht groß vorbereitet, habe gegen einen Mann kandidiert und hatte dann eine Stimme mehr und war Kandidatin. Dem anderen Kandidaten blieb die Spucke weg und mir auch. Wenn ich an den Wahlkampf denke, ohne Ausstattung, ohne Mittel und gerade geschieden... war eigentlich mehr mein Schwerpunkt als Familienpolitik. Die FDP in Baden-Württemberg war ja auch immer Bürgerrechtspartei und wir haben ab 1996 dort den Justizminister gestellt. Ich bin dann 2002 auch in den Rechtsausschuss des Bundestages gekommen. Ich sage es offen - das war sehr nüchtern. Ich habe damals die Reform des Betreuungsrechts bearbeitet und Erfahrungen gesammelt, wie man als Abgeordnete arbeitet. Das Betreuungsrecht war eine spannende Aufgabenstellung. Ich habe damals immer die These vertreten, dass eine psychische Erkrankung nicht bedeuten muss, dass der Mensch eingesperrt wird. Ich denke oft in diesem Zusammenhang an den aktuellen Fall in München. Ich will 
der Entscheidung dort nicht vorgreifen, aber der Fall erinnert mich an diese Arbeitszeit und diese ersten Schritte als Abgeordnete. Ich habe dann gemerkt, dass die Thematik der Gleichstellungspolitik im Rechtsausschuss überhaupt keinen Boden hat. Das hat mich aber interessiert, es ist auch mein Hintergrund. Ich habe während meines Studiums in Heidelberg die Frauenbewegung hautnah erlebt und ich wollte auch in diesem Feld weiter aktiv sein. Deshalb habe ich mich dann entschieden, in den Familienausschuss zu wechseln und habe die Stellvertreterposition im Rechtsausschuss beibehalten. Ich habe mich also thematisch stark umorientiert, was eher untypisch ist. Auch unter Karrieregesichtspunkten ist ein solcher Wechsel nicht optimal. Das hat mich aber einfach mehr interessiert und ich habe in dem Themenbereich auch mehr Entwicklungsmöglichkeiten gefunden. Ich war dann integrationspolitische Sprecherin meiner Fraktion. Die gesellschaftspolitischen Fragen von Gleichstellung, Emanzipation von Männern, Frauen, In- und Ausländern, Menschen mit und ohne Behinderung, von Jung und Alt, die sind im Familienausschuss richtig angesiedelt und bräuchten eine stärkere Stimme. Ich habe mich in diesem Bereich engagiert gezeigt und das ist möglicherweise auch ein Grund gewesen, warum man mir 2009 den Ausschussvorsitz angeboten hat.

\section{Sie kandidieren nicht erneut für den Bundestag, was bringt die Zukunft für Sie?}

Das was die Vergangenheit auch schon war - weiterhin als Anwältin zu arbeiten. Das habe ich immer als eine gute Erdung empfunden, den praktischen Zugang zu den Themen zu haben, die uns im Bundestag bewegen. Es ist schon auch ein Unterschied, nicht alle Kolleginnen und Kollegen haben das und ich halte es für ganz wesentlich, eine berufspraktische Perspektive in die politische Arbeit einzubringen. Deshalb habe ich mich auch damals den Klägern einer Organklage vor dem Bundesverfassungsgericht gegen die Veröffentlichungspflicht der Nebeneinkünfte von Abgeordneten angeschlossen, weil ich der Meinung bin, dass die Entscheidung darauf abzielt, es für Selbständige unattraktiv zu machen, in die politische Arbeit zu gehen. Selbständige gehen ja ohnehin schon ein großes Risiko ein, wenn sie in die Politik bzw. in den Bundestag gehen. Wenn sie dann noch offenlegen müssen, was sie sozusagen nebenher verdienen, dann ist das langfristig darauf angelegt, diese Berufe aus der Politik zu verdrängen. Das Bundesverfassungsgericht hat sich nicht mehrheitlich für diese Position entscheiden können. Es war ein Patt. Ich habe aber während der Verhandlungen gemerkt, wie die Nachdenklichkeit stieg. Ich sehe auch jetzt die Entwicklung, es kommen immer weniger Leute mit einem lebenspraktischen Hintergrund ins Parlament.

Was die Parteiorganisation angeht, kann man ja sagen, das ist nicht so attraktiv für Frauen als Berufsfeld.

Ja. Die Parteien haben einen verfassungsrechtlichen Auftrag, sie wirken an der Meinungsbildung mit, aber sie sind auch limitiert. Parteien organisieren die Macht und gleichzeitig sind sie auch ein Feld für sehr anerkennungsbedürftige Men- schen. Das schafft Abhängigkeiten. Das ist für Frauen auch ein schwieriges Feld. Frauen werden da gerne verheizt und kommen dann eben nicht weiter.

\section{Sie haben nach einer kontroversen Debatte gegen ihre Frak- tion für die Frauenquote gestimmt. Warum?}

Ich muss gestehen, ich bin gar nicht immer eine heftige Befürworterin der Quote gewesen. Das ist ja das Selbstverständnis der meisten Kolleginnen, durch Leistung zu überzeugen und nicht durch Quote. Die Diskussion ist aber immer stärker auch von Europa und von Brüssel beflügelt worden und ich habe mich dann natürlich mit dem Thema Quote auseinandergesetzt. Auch die Aktivitäten des Juristinnenbundes habe ich als Mitglied natürlich verfolgt, wie fundiert juristisch da argumentiert wird. Ich stand also dem Thema Quote immer offener gegenüber, ganz sicher auch aus der parteipolitischen Erfahrung heraus. Frauen sind in allen Parteien, in denen es keine Quote gibt, einfach unterrepräsentiert. Das hängt nicht mit Können zusammen, sondern mit indirekten Mechanismen. Die Frauenförderung ist eine Aufgabe der Gesellschaft und wir sind nur zukunftsfähig, wenn wir Frauen auch tatsächlich gleichstellen und nicht nur rechtlich. Ich habe dann als einige der wenigen FDP Frauen hier in der Fraktion die Berliner Erklärung unterschrieben. Es sah auch eine Weile so aus, als könnte ein überfraktioneller Antrag gelingen, auch aus den Bundesländern kamen gute Signale. Dann kommt es schon zum Schwur - in welcher Fraktion ist was möglich. Die Diskussion in der Union hat dann dazu geführt, dass die Quote nun zwar im Wahlprogramm steht, aber „man“ sich bei der Abstimmung zurückgehalten hat. Plötzlich war ich dann die einzige Abweichlerin im Regierungslager. Ich habe das meiner Fraktionsführung mitgeteilt und gesagt, dass das nach meinem politischen Lebensweg eine Überzeugungsfrage ist. Ich war es mir selber schuldig, dass ich das durchziehe. Man war zwar nicht begeistert, aber man hat mich machen lassen. Fraktionszwang habe ich also nicht gespürt. Es hat dann ja noch einige Medienaufmerksamkeit gegeben für meine Position. Es gibt zwar immer mal wieder Themen, bei denen Kollegen anders abstimmen. Aber wenn es eine Frau macht, dann ist das besonders kritisch. Das war schon auch der Punkt dabei, es fiel sehr auf, dass eine Frau das durchhält. Da war aber auch meine kommunalpolitische Erfahrung hilfreich, wo man auch sehr in der Öffentlichkeit steht und wo ich auch schon mehr als eine Position kontrovers vertreten habe.

\section{Haben Sie sich gefreut, als das Bundesverfassungsgericht das Ehegattensplitting auch für gleichgeschlechtliche Paare öffnete?}

Ja, das war ja abzusehen, dass es in diese Richtung geht und es ist auch richtig, in unserer modernen Gesellschaft nicht mehr ausschließlich an der Ehe anzuknüpfen. Das zeigt einfach, dass wir auch gesellschaftlich in einem Umbruch sind. Die ganze Vielfalt in der Gesellschaft sollte gleiche Chancen erhalten. 
Was hätten Sie in ihrer aktiven Zeit als Bundespolitikerin noch gern erlebt?

Ich wünsche mir für zwei Themen mehr Aufmerksamkeit der Kampf gegen die Gewalt in unserer Gesellschaft und ein größeres Augenmerk auf Kinder und Jugendliche. Wir brauchen eine klarere Aufstellung gegen Gewalt in der Familie und solide finanzierte Beratung, Frauenhäuser, aber auch Beratung und Hilfe für die Opfer von Kindesmissbrauch als einer besonderen Form von Gewalt in der Familie. Die Finanzierung solcher Angebote ist auch ein verfassungsrechtliches Problem und sollte in einer Föderalismusreform angegangen werden. Und das Augenmerk auf Kinder und Jugendliche muss geschärft werden. Ich habe gerade erst ein Familiengericht erlebt, das ganz stark mit Verweis auf das Grundgesetz das Recht der Eltern in den Mittelpunkt gestellt hat. Ich finde, Kinderrechte gehören ins Grundgesetz, damit man auch mit dem Recht des Kindes argumentieren kann. Natürlich stehen alle Menschen im Grundgesetz, aber der besondere Blick auf das besonders empfindsame und schützenswerte Kind bzw. Jugendliche scheint mir eine Aufgabenstellung für die Zukunft zu sein, an der ich jetzt nicht mehr parlamentarisch arbeiten kann, aber wofür ich natürlich eine Botschaft habe.

Haben Sie einen Karrieretipp für junge Juristinnen, die sich heute in einer Männerwelt behaupten wollen?

Sich nicht um das Thema Männer scheren, sondern ihr eigenes Ding machen, ihr eigenes Thema finden. Es gibt dann auch Anfeindungen, aber man verschafft sich auch Respekt, wenn man möglichst unbefangen ist und die eigenen Überzeugungen vertritt. Und was ich Juristinnen immer empfehle - wirtschaftliche Zusammenhänge verstehen.

\section{Aktuelle Pressemitteilungen und Stellungnahmen}

\section{Pressemitteilungen \\ 13-21 Endspurt: djb-Projekt „Aktionärinnen fordern Gleichberechtigung - 2013“ geht in die letzte Runde $(1.8 .2013)$}

13-20 djb fordert von litauischer Ratspräsidentschaft, die Überarbeitung der Mutterschutzrichtlinie fortzusetzen (16.7.2013)

13-19 Aktionärinnen fordern Gleichberechtigung: Keine Schonzeit für Fielmann (11.7.2013)

13-18 djb gratuliert: Bayerischer Verdienstorden für Renate Maltry (1.7.2013)

13-17 Für eine Reform der Minijobs (Gemeinsamer Aufruf zahlreicher Verbände) (25.6.2013)

13-16 djb fordert Individualbesteuerung (7.6.2013)

13-15 Zurück auf Null: Luisa Deplazes Delgado verlässt SAP-Vorstand (4.6.2013)

13-14 Vertrauliche Geburt - djb fordert gesetzliche Regelung noch vor der Wahl (23.5.2013)

13-13 Bundestagsaktion „Spitzenfrauen fragen Spitzenkandidaten “: Steinbrück verspricht eine 40 ProzentVorstandsquote bis 2017 / Von der Leyen: „Das Land braucht die Quote“ (17.5.2013)

13-12 „Spitzenfrauen fragen Spitzenkandidaten“ am 17. Mai in Berlin. Steinbrück, von der Leyen, Wagenknecht, Göring-Eckardt und Kolb antworten Frauenverbänden (17.5.2013)

13-11 „Nein“ zur Frauenquote für die Aufsichtsräte - Gesetzgeber ignoriert Verfassungsauftrag (19.4.2013)

13-10 Auf der Zielgeraden ist ein klares Bekenntnis gefragt“ - Frauenverbände fordern ein Votum für Quote in Aufsichtsräten - Verbände verlangen, den Fraktionszwang aufzuheben und eine freie Abstimmung zuzulassen (15. April 2013)
13-09 djb zu fünf häufigen Irrtümern über das Ehegattensplitting (Anhang: 13-09E Erklärung: Zu den fünf häufigsten Irrtümern über das Ehegattensplitting) (22.3.2013)

13-08 djb-Initiative „Frauen in die Roten Roben“ hat durchschlagenden Erfolg (21.3.2013)

13-07 Massive Verschlechterungen bei der Prozesskostenhilfe benachteiligen Alleinerziehende (Gemeinsame Pressemitteilung zahlreicher Verbände) (13.3.2013)

13-06 djb fordert Einsatz qualifizierter Dolmetscherinnen und Dolmetscher in Strafverfahren - auch für die Opfer der Straftaten (8.3.2013)

13-05 Schutz der „Altehe“ - Mitnichten eine Reform des Unterhaltsrechts (28.2.2013)

13-04 Mehr Gleichbehandlung für eingetragene Lebenspartnerschaften: Bundesverfassungsgericht bestätigt auch bei den Sukzessivadoptionen die Position des Deutschen Juristinnenbundes (19.2.2013)

13-03 Superwahljahr für die Aufsichtsräte - super Wahljahr auch für Frauen? (18.1.2013)

13-02 Quote für Aufsichtsräte und Vorstände rechtlich geboten (17.1.2013)

13-01 Gender Pay Gap lässt sich mit statistischen Tricks nicht „wegrechnen“ (16.1.2013)

\section{Stellungnahmen}

13-07 Stellungnahme zum Vorschlag für eine Richtlinie des Europäischen Parlaments und des Rates zur Änderung der Richtlinie 92/85/EWG des Rates vom 19. Oktober 1992 über die Durchführung von Maßnahmen zur Verbesserung der Sicherheit und des Gesundheitsschutzes von schwangeren Arbeitnehmerinnen, Wöchnerinnen und stillenden Arbeitnehmerinnen am Arbeitsplatz, KOM(2008)637endg. (16.7.2013) 